\title{
Measurement of the Forward-Backward Charge Asymmetry in Top-Quark Pair Production
}

V. M. Abazov, ${ }^{36}$ B. Abbott, ${ }^{76}$ M. Abolins,${ }^{66}$ B. S. Acharya,${ }^{29}$ M. Adams, ${ }^{52}$ T. Adams,${ }^{50}$ E. Aguilo, ${ }^{6}$ S. H. Ahn, ${ }^{31}$ M. Ahsan, ${ }^{60}$ G. D. Alexeev, ${ }^{36}$ G. Alkhazov, ${ }^{40}$ A. Alton, ${ }^{65}$.* G. Alverson, ${ }^{64}$ G. A. Alves, ${ }^{2}$ M. Anastasoaie,${ }^{35}$ L. S. Ancu, ${ }^{35}$ T. Andeen,${ }^{54}$ S. Anderson, ${ }^{46}$ B. Andrieu, ${ }^{17}$ M. S. Anzelc, ${ }^{54}$ Y. Arnoud, ${ }^{14}$ M. Arov, ${ }^{61}$ M. Arthaud,${ }^{18}$ A. Askew, ${ }^{50}$ B. Asman, ${ }^{41}$ A. C. S. Assis Jesus, ${ }^{3}$ O. Atramentov, ${ }^{50}$ C. Autermann, ${ }^{21}$ C. Avila, ${ }^{8}$ C. Ay, ${ }^{24}$ F. Badaud, ${ }^{13}$ A. Baden, ${ }^{62}$ L. Bagby, ${ }^{53}$ B. Baldin, ${ }^{51}$ D. V. Bandurin, ${ }^{60}$ S. Banerjee, ${ }^{29}$ P. Banerjee,${ }^{29}$ E. Barberis ${ }^{64}$ A.-F. Barfuss,${ }^{15}$ P. Bargassa ${ }^{81}$ P. Baringer, ${ }^{59}$ J. Barreto, ${ }^{2}$ J. F. Bartlett,${ }^{51}$ U. Bassler, ${ }^{18}$ D. Bauer, ${ }^{44}$ S. Beale, ${ }^{6}$ A. Bean,${ }^{59}$ M. Begalli, ${ }^{3}$ M. Begel,${ }^{72}$ C. Belanger-Champagne, ${ }^{41}$ L. Bellantoni, ${ }^{51}$ A. Bellavance, ${ }^{51}$ J. A. Benitez ${ }^{66}$ S. B. Beri, ${ }^{27}$ G. Bernardi, ${ }^{17}$ R. Bernhard, ${ }^{23}$ I. Bertram, ${ }^{43}$ M. Besançon, ${ }^{18}$ R. Beuselinck ${ }^{44}$ V. A. Bezzubov, ${ }^{39}$ P. C. Bhat,${ }^{51}$ V. Bhatnagar, ${ }^{27}$ C. Biscarat,${ }^{20}$ G. Blazey, ${ }^{53}$ F. Blekman ${ }^{44}$ S. Blessing, ${ }^{50}$ D. Bloch,${ }^{19}$ K. Bloom, ${ }^{68}$ A. Boehnlein, ${ }^{51}$ D. Boline, ${ }^{63}$ T. A. Bolton,${ }^{60}$ G. Borissov, ${ }^{43}$ T. Bose, ${ }^{78}$ A. Brandt, ${ }^{79}$ R. Brock, ${ }^{66}$ G. Brooijmans, ${ }^{71}$ A. Bross, ${ }^{51}$ D. Brown, ${ }^{82}$ N. J. Buchanan, ${ }^{50}$ D. Buchholz,${ }^{54}$ M. Buehler, ${ }^{82}$ V. Buescher, ${ }^{22}$ V. Bunichev, ${ }^{38}$ S. Burdin, ${ }^{43, \dagger}$ S. Burke, ${ }^{46}$ T. H. Burnett, ${ }^{83}$ C. P. Buszello, ${ }^{44}$ J. M. Butler, ${ }^{63}$ P. Calfayan, ${ }^{25}$ S. Calvet, ${ }^{16}$ J. Cammin, ${ }^{72}$ W. Carvalho, ${ }^{3}$ B. C. K. Casey,${ }^{51}$ N. M. Cason, ${ }^{56}$ H. Castilla-Valdez, ${ }^{33}$ S. Chakrabarti, ${ }^{18}$ D. Chakraborty, ${ }^{53}$ K. M. Chan,${ }^{56}$ K. Chan, ${ }^{6}$ A. Chandra, ${ }^{49}$ F. Charles, ${ }^{19,}$ E. Cheu, ${ }^{46}$ F. Chevallier, ${ }^{14}$ D. K. Cho, ${ }^{63}$ S. Choi,${ }^{32}$ B. Choudhary, ${ }^{28}$ L. Christofek, ${ }^{78}$ T. Christoudias, ${ }^{44}$ S. Cihangir, ${ }^{51}$ D. Claes, ${ }^{68}$ Y. Coadou, ${ }^{6}$ M. Cooke, ${ }^{81}$ W. E. Cooper, ${ }^{51}$ M. Corcoran, ${ }^{81}$ F. Couderc, ${ }^{18}$ M.-C. Cousinou, ${ }^{15}$ S. Crépé-Renaudin,,${ }^{14}$ D. Cutts, ${ }^{78}$ M. Ćwiok, ${ }^{30}$ H. da Motta, ${ }^{2}$ A. Das,${ }^{46}$ G. Davies,${ }^{44}$ K. De ${ }^{79}$ S. J. de Jong,${ }^{35}$ E. De La Cruz-Burelo, ${ }^{65}$

C. De Oliveira Martins, ${ }^{3}$ J. D. Degenhardt, ${ }^{65}$ F. Déliot, ${ }^{18}$ M. Demarteau,${ }^{51}$ R. Demina, ${ }^{72}$ D. Denisov,${ }^{51}$ S. P. Denisov ${ }^{39}$ S. Desai, ${ }^{51}$ H. T. Diehl, ${ }^{51}$ M. Diesburg, ${ }^{51}$ A. Dominguez, ${ }^{68}$ H. Dong, ${ }^{73}$ L. V. Dudko, ${ }^{38}$ L. Duflot, ${ }^{16}$ S. R. Dugad, ${ }^{29}$ D. Duggan, ${ }^{50}$ A. Duperrin, ${ }^{15}$ J. Dyer, ${ }^{66}$ A. Dyshkant, ${ }^{53}$ M. Eads, ${ }^{68}$ D. Edmunds, ${ }^{66}$ J. Ellison, ${ }^{49}$ V. D. Elvira, ${ }^{51}$ Y. Enari, ${ }^{78}$ S. Eno, ${ }^{62}$ P. Ermolov, ${ }^{38}$ H. Evans, ${ }^{55}$ A. Evdokimov, ${ }^{74}$ V. N. Evdokimov, ${ }^{39}$ A. V. Ferapontov, ${ }^{60}$ T. Ferbel, ${ }^{72}$ F. Fiedler, ${ }^{24}$ F. Filthaut, ${ }^{35}$ W. Fisher,${ }^{51}$ H. E. Fisk, ${ }^{51}$ M. Ford,${ }^{45}$ M. Fortner, ${ }^{53}$ H. Fox ${ }^{23}$ S. Fu,${ }^{51}$ S. Fuess, ${ }^{51}$ T. Gadfort,${ }^{83}$ C. F. Galea, ${ }^{35}$ E. Gallas, ${ }^{51}$ E. Galyaev ${ }^{56}$ C. Garcia, ${ }^{72}$ A. Garcia-Bellido, ${ }^{83}$ V. Gavrilov,${ }^{37}$ P. Gay, ${ }^{13}$ W. Geist, ${ }^{19}$ D. Gelé, ${ }^{19}$ C. E. Gerber,${ }^{52}$ Y. Gershtein, ${ }^{50}$ D. Gillberg, ${ }^{6}$ G. Ginther, ${ }^{72}$ N. Gollub, ${ }^{41}$ B. Gómez, ${ }^{8}$ A. Goussiou,${ }^{56}$ P. D. Grannis,${ }^{73}$ H. Greenlee ${ }^{51}$ Z. D. Greenwood, ${ }^{61}$ E. M. Gregores, ${ }^{4}$ G. Grenier,${ }^{20}$ Ph. Gris, ${ }^{13}$ J.-F. Grivaz, ${ }^{16}$ A. Grohsjean, ${ }^{25}$ S. Grünendahl,${ }^{51}$ M. W. Grünewald ${ }^{30}$ J. Guo, ${ }^{73}$ F. Guo, ${ }^{73}$ P. Gutierrez, ${ }^{76}$ G. Gutierrez, ${ }^{51}$ A. Haas, ${ }^{71}$ N. J. Hadley, ${ }^{62}$ P. Haefner,${ }^{25}$ S. Hagopian, ${ }^{50}$ J. Haley, ${ }^{69}$ I. Hall,${ }^{66}$ R. E. Hall, ${ }^{48}$ L. Han, ${ }^{7}$ K. Hanagaki, ${ }^{51}$ P. Hansson, ${ }^{41}$ K. Harder, ${ }^{45}$ A. Harel, ${ }^{72}$ R. Harrington, ${ }^{64}$ J. M. Hauptman, ${ }^{58}$ R. Hauser, ${ }^{66}$ J. Hays,${ }^{44}$ T. Hebbeker,${ }^{21}$ D. Hedin, ${ }^{53}$ J. G. Hegeman, ${ }^{34}$ J. M. Heinmiller, ${ }^{52}$ A. P. Heinson, ${ }^{49}$ U. Heintz, ${ }^{63}$ C. Hensel, ${ }^{59}$ K. Herner,${ }^{73}$ G. Hesketh,${ }^{64}$ M. D. Hildreth,${ }^{56}$ R. Hirosky, ${ }^{82}$ J. D. Hobbs, ${ }^{73}$ B. Hoeneisen, ${ }^{12}$ H. Hoeth ${ }^{26}$ M. Hohlfeld, ${ }^{22}$ S. J. Hong ${ }^{31}$ S. Hossain, ${ }^{76}$ P. Houben, ${ }^{34}$ Y. Hu, ${ }^{73}$ Z. Hubacek, ${ }^{10}$ V. Hynek, ${ }^{9}$ I. Iashvili, ${ }^{70}$ R. Illingworth,${ }^{51}$ A. S. Ito, ${ }^{51}$ S. Jabeen, ${ }^{63}$ M. Jaffré, ${ }^{16}$ S. Jain, ${ }^{76}$ K. Jakobs,${ }^{23}$ C. Jarvis, ${ }^{62}$ R. Jesik, ${ }^{44}$ K. Johns, ${ }^{46}$ C. Johnson, ${ }^{71}$ M. Johnson, ${ }^{51}$ A. Jonckheere, ${ }^{51}$ P. Jonsson, ${ }^{44}$ A. Juste, ${ }^{51}$ D. Käfer ${ }^{21}$ E. Kajfasz, ${ }^{15}$ A. M. Kalinin, ${ }^{36}$ J. R. Kalk, ${ }^{66}$ J. M. Kalk, ${ }^{61}$ S. Kappler, ${ }^{21}$ D. Karmanov, ${ }^{38}$ P. Kasper,${ }^{51}$ I. Katsanos, ${ }^{71}$ D. Kau, ${ }^{50}$ R. Kaur, ${ }^{27}$

V. Kaushik, ${ }^{79}$ R. Kehoe, ${ }^{80}$ S. Kermiche, ${ }^{15}$ N. Khalatyan, ${ }^{51}$ A. Khanov, ${ }^{77}$ A. Kharchilava, ${ }^{70}$ Y. M. Kharzheev, ${ }^{36}$ D. Khatidze, ${ }^{71}$ H. Kim, ${ }^{32}$ T. J. Kim, ${ }^{31}$ M. H. Kirby, ${ }^{54}$ M. Kirsch, ${ }^{21}$ B. Klima, ${ }^{51}$ J. M. Kohli, ${ }^{27}$ J.-P. Konrath, ${ }^{23}$ M. Kopal, ${ }^{76}$ V. M. Korablev, ${ }^{39}$ A. V. Kozelov, ${ }^{39}$ D. Krop, ${ }^{55}$ T. Kuhl, ${ }^{24}$ A. Kumar, ${ }^{70}$ S. Kunori, ${ }^{62}$ A. Kupco, ${ }^{11}$ T. Kurča, ${ }^{20}$ J. Kvita, ${ }^{9}$ F. Lacroix, ${ }^{13}$ D. Lam, ${ }^{56}$ S. Lammers, ${ }^{71}$ G. Landsberg, ${ }^{78}$ P. Lebrun, ${ }^{20}$ W. M. Lee,${ }^{51}$ A. Leflat, ${ }^{38}$ F. Lehner, ${ }^{42}$ J. Lellouch, ${ }^{17}$ J. Leveque, ${ }^{46}$ P. Lewis, ${ }^{44}$ J. Li, ${ }^{79}$ Q. Z. Li,${ }^{51}$ L. Li, ${ }^{49}$ S. M. Lietti, ${ }^{5}$ J. G. R. Lima ${ }^{53}$ D. Lincoln,,${ }^{51}$ J. Linnemann, ${ }^{66}$ V. V. Lipaev,${ }^{39}$ R. Lipton, ${ }^{51}$ Y. Liu, ${ }^{7}$ Z. Liu, ${ }^{6}$ L. Lobo,${ }^{44}$ A. Lobodenko, ${ }^{40}$ M. Lokajicek, ${ }^{11}$ P. Love, ${ }^{43}$ H. J. Lubatti, ${ }^{83}$ A. L. Lyon, ${ }^{51}$ A. K. A. Maciel, ${ }^{2}$ D. Mackin, ${ }^{81}$ R. J. Madaras, ${ }^{47}$ P. Mättig, ${ }^{26}$ C. Magass, ${ }^{21}$ A. Magerkurth,${ }^{65}$ P. K. Mal, ${ }^{56}$ H. B. Malbouisson, ${ }^{3}$ S. Malik, ${ }^{68}$ V. L. Malyshev, ${ }^{36}$ H. S. Mao, ${ }^{51}$ Y. Maravin, ${ }^{60}$ B. Martin, ${ }^{14}$ R. McCarthy, ${ }^{73}$ A. Melnitchouk, ${ }^{67}$ A. Mendes, ${ }^{15}$ L. Mendoza, ${ }^{8}$ P. G. Mercadante, ${ }^{5}$ M. Merkin, ${ }^{38}$ K. W. Merritt, ${ }^{51}$ J. Meyer, ${ }^{22,}$ A. Meyer, $^{21}$ T. Millet, ${ }^{20}$ J. Mitrevski, ${ }^{71}$ J. Molina, ${ }^{3}$ R. K. Mommsen, ${ }^{45}$ N. K. Mondal, ${ }^{29}$ R. W. Moore, ${ }^{6}$ T. Moulik, ${ }^{59}$ G. S. Muanza, ${ }^{20}$ M. Mulders ${ }^{51}$ M. Mulhearn, ${ }^{71}$ O. Mundal, ${ }^{22}$ L. Mundim, ${ }^{3}$ E. Nagy, ${ }^{15}$ M. Naimuddin, ${ }^{51}$ M. Narain, ${ }^{78}$ N. A. Naumann, ${ }^{35}$ H. A. Neal, ${ }^{65}$ J. P. Negret, ${ }^{8}$ P. Neustroev,${ }^{40}$ H. Nilsen, ${ }^{23}$ H. Nogima, ${ }^{3}$ A. Nomerotski, ${ }^{51}$ S. F. Novaes, ${ }^{5}$ T. Nunnemann, ${ }^{25}$ V. O’Dell, ${ }^{51}$ D. C. O'Neil, ${ }^{6}$ G. Obrant, ${ }^{40}$ C. Ochando, ${ }^{16}$ D. Onoprienko, ${ }^{60}$ N. Oshima, ${ }^{51}$ J. Osta, ${ }^{56}$ R. Otec, ${ }^{10}$ G. J. Otero y Garzón, ${ }^{51}$ M. Owen, ${ }^{45}$ P. Padley, ${ }^{81}$ M. Pangilinan, ${ }^{78}$ N. Parashar, ${ }^{57}$ S.-J. Park, ${ }^{72}$ S. K. Park, ${ }^{31}$ J. Parsons,${ }^{71}$ R. Partridge,${ }^{78}$ N. Parua,${ }^{55}$ A. Patwa,${ }^{74}$ G. Pawloski,${ }^{81}$ B. Penning, ${ }^{23}$ M. Perfilov, ${ }^{38}$ K. Peters,${ }^{45}$ Y. Peters, ${ }^{26}$ P. Pétroff,${ }^{16}$ M. Petteni, ${ }^{44}$ R. Piegaia, ${ }^{1}$ J. Piper, ${ }^{66}$ M.-A. Pleier, ${ }^{22}$ P. L. M. Podesta-Lerma, ${ }^{33, \|}$ V. M. Podstavkov, ${ }^{51}$ Y. Pogorelov, ${ }^{56}$ 
M.-E. Pol, ${ }^{2}$ P. Polozov,${ }^{37}$ B. G. Pope,${ }^{66}$ A. V. Popov,${ }^{39}$ C. Potter,${ }^{6}$ W. L. Prado da Silva, ${ }^{3}$ H. B. Prosper, ${ }^{50}$ S. Protopopescu, ${ }^{74}$ J. Qian, ${ }^{65}$ A. Quadt, ${ }^{22,8}$ B. Quinn, ${ }^{67}$ A. Rakitine, ${ }^{43}$ M. S. Rangel,,${ }^{2}$ K. Ranjan, ${ }^{28}$ P. N. Ratoff,,${ }^{43}$ P. Renkel, ${ }^{80}$ S. Reucroft,${ }^{64}$ P. Rich, ${ }^{45}$ M. Rijssenbeek, ${ }^{73}$ I. Ripp-Baudot, ${ }^{19}$ F. Rizatdinova, ${ }^{77}$ S. Robinson, ${ }^{44}$ R. F. Rodrigues, ${ }^{3}$ M. Rominsky, ${ }^{76}$ C. Royon, ${ }^{18}$ P. Rubinov, ${ }^{51}$ R. Ruchti, ${ }^{56}$ G. Safronov, ${ }^{37}$ G. Sajot, ${ }^{14}$ A. Sánchez-Hernández,${ }^{33}$ M. P. Sanders, ${ }^{17}$ A. Santoro, ${ }^{3}$ G. Savage, ${ }^{51}$ L. Sawyer, ${ }^{61}$ T. Scanlon, ${ }^{44}$ D. Schaile, ${ }^{25}$ R. D. Schamberger ${ }^{73}$ Y. Scheglov, ${ }^{40}$ H. Schellman, ${ }^{54}$ P. Schieferdecker, ${ }^{25}$ T. Schliephake, ${ }^{26}$ C. Schwanenberger, ${ }^{45}$ A. Schwartzman, ${ }^{69}$ R. Schwienhorst, ${ }^{66}$ J. Sekaric, ${ }^{50}$ H. Severini, ${ }^{76}$ E. Shabalina, ${ }^{52}$ M. Shamim, ${ }^{60}$ V. Shary, ${ }^{18}$ A. A. Shchukin, ${ }^{39}$ R. K. Shivpuri, ${ }^{28}$ V. Siccardi, ${ }^{19}$ V. Simak, ${ }^{10}$ V. Sirotenko, ${ }^{51}$ P. Skubic,${ }^{76}$ P. Slattery, ${ }^{72}$ D. Smirnov, ${ }^{56}$ J. Snow, ${ }^{75}$ G. R. Snow, ${ }^{68}$ S. Snyder, ${ }^{74}$ S. Söldner-Rembold ${ }^{45}$ L. Sonnenschein, ${ }^{17}$ A. Sopczak, ${ }^{43}$ M. Sosebee ${ }^{79}$ K. Soustruznik, ${ }^{9}$ M. Souza, ${ }^{2}$ B. Spurlock, ${ }^{79}$ J. Stark,${ }^{14}$ J. Steele, ${ }^{61}$ V. Stolin, ${ }^{37}$ D. A. Stoyanova, ${ }^{39}$ J. Strandberg,${ }^{65}$ S. Strandberg,,${ }^{41}$ M. A. Strang, ${ }^{70}$ M. Strauss,${ }^{76}$ E. Strauss,${ }^{73}$ R. Ströhmer,${ }^{25}$ D. Strom,${ }^{54}$ L. Stutte ${ }^{51}$ S. Sumowidagdo ${ }^{50}$ P. Svoisky, ${ }^{56}$ A. Sznajder, ${ }^{3}$ M. Talby, ${ }^{15}$ P. Tamburello, ${ }^{46}$ A. Tanasijczuk, ${ }^{1}$ W. Taylor, ${ }^{6}$ J. Temple, ${ }^{46}$ B. Tiller,${ }^{25}$ F. Tissandier,${ }^{13}$ M. Titov, ${ }^{18}$ V. V. Tokmenin,${ }^{36}$ T. Toole,${ }^{62}$ I. Torchiani,${ }^{23}$ T. Trefzger, ${ }^{24}$ D. Tsybychev, ${ }^{73}$ B. Tuchming, ${ }^{18}$ C. Tully,${ }^{69}$ P. M. Tuts, ${ }^{71}$ R. Unalan, ${ }^{66}$ S. Uvarov,${ }^{40}$ L. Uvarov,${ }^{40}$ S. Uzunyan,${ }^{53}$ B. Vachon, ${ }^{6}$ P. J. van den Berg, ${ }^{34}$ R. Van Kooten,${ }^{55}$ W. M. van Leeuwen, ${ }^{34}$ N. Varelas, ${ }^{52}$ E. W. Varnes, ${ }^{46}$ I. A. Vasilyev, ${ }^{39}$ M. Vaupel, ${ }^{26}$

P. Verdier, ${ }^{20}$ L. S. Vertogradov ${ }^{36}$ M. Verzocchi, ${ }^{51}$ F. Villeneuve-Seguier, ${ }^{44}$ P. Vint, ${ }^{44}$ P. Vokac, ${ }^{10}$ E. Von Toerne, ${ }^{60}$ M. Voutilainen, ${ }^{68, \pi}$ R. Wagner, ${ }^{69}$ H. D. Wahl,${ }^{50}$ L. Wang, ${ }^{62}$ M. H. L. S Wang, ${ }^{51}$ J. Warchol, ${ }^{56}$ G. Watts, ${ }^{83}$ M. Wayne, ${ }^{56}$ M. Weber, ${ }^{51}$ G. Weber, ${ }^{24}$ A. Wenger $,{ }^{23}, * *$ N. Wermes, ${ }^{22}$ M. Wetstein, ${ }^{62}$ A. White, ${ }^{79}$ D. Wicke, ${ }^{26}$ G. W. Wilson, ${ }^{59}$ S. J. Wimpenny, ${ }^{49}$ M. Wobisch, ${ }^{61}$ D. R. Wood, ${ }^{64}$ T. R. Wyatt, ${ }^{45}$ Y. Xie,${ }^{78}$ S. Yacoob,${ }^{54}$ R. Yamada,${ }^{51}$ M. Yan,${ }^{62}$ T. Yasuda,${ }^{51}$ Y. A. Yatsunenko, ${ }^{36}$ K. Yip,${ }^{74}$ H. D. Yoo ${ }^{78}$ S. W. Youn,${ }^{54}$ J. Yu, ${ }^{79}$ A. Zatserklyaniy, ${ }^{53}$ C. Zeitnitz ${ }^{26}$ T. Zhao, ${ }^{83}$ B. Zhou, ${ }^{65}$ J. Zhu, ${ }^{73}$ M. Zielinski, ${ }^{72}$ D. Zieminska, ${ }^{55}$ A. Zieminski, ${ }^{55,}$ L. Zivkovic, ${ }^{71}$ V. Zutshi, ${ }^{53}$ and E. G. Zverev ${ }^{38}$

\title{
(D0 Collaboration)
}

\author{
${ }^{1}$ Universidad de Buenos Aires, Buenos Aires, Argentina \\ ${ }^{2}$ LAFEX, Centro Brasileiro de Pesquisas Físicas, Rio de Janeiro, Brazil \\ ${ }^{3}$ Universidade do Estado do Rio de Janeiro, Rio de Janeiro, Brazil \\ ${ }^{4}$ Universidade Federal do ABC, Santo André, Brazil \\ ${ }^{5}$ Instituto de Física Teórica, Universidade Estadual Paulista, São Paulo, Brazil \\ ${ }^{6}$ University of Alberta, Edmonton, Alberta, Canada, \\ Simon Fraser University, Burnaby, British Columbia, Canada, \\ York University, Toronto, Ontario, Canada, \\ and McGill University, Montreal, Quebec, Canada \\ ${ }^{7}$ University of Science and Technology of China, Hefei, People's Republic of China \\ ${ }^{8}$ Universidad de los Andes, Bogotá, Colombia \\ ${ }^{9}$ Center for Particle Physics, Charles University, Prague, Czech Republic \\ ${ }^{10}$ Czech Technical University, Prague, Czech Republic \\ ${ }^{11}$ Center for Particle Physics, Institute of Physics, Academy of Sciences of the Czech Republic, Prague, Czech Republic \\ ${ }^{12}$ Universidad San Francisco de Quito, Quito, Ecuador \\ ${ }^{13}$ Laboratoire de Physique Corpusculaire, IN2P3-CNRS, Université Blaise Pascal, Clermont-Ferrand, France \\ ${ }^{14}$ Laboratoire de Physique Subatomique et de Cosmologie, IN2P3-CNRS, Universite de Grenoble 1, Grenoble, France \\ ${ }^{15}$ CPPM, IN2P3-CNRS, Université de la Méditerranée, Marseille, France \\ ${ }^{16}$ Laboratoire de l'Accélérateur Linéaire, IN2P3-CNRS et Université Paris-Sud, Orsay, France \\ ${ }^{17}$ LPNHE, IN2P3-CNRS, Universités Paris VI and VII, Paris, France \\ ${ }^{18}$ DAPNIA/Service de Physique des Particules, CEA, Saclay, France \\ ${ }^{19}$ IPHC, Université Louis Pasteur et Université de Haute Alsace, CNRS, IN2P3, Strasbourg, France \\ ${ }^{20}$ IPNL, Université Lyon 1, CNRS/IN2P3, Villeurbanne, France \\ and Université de Lyon, Lyon, France \\ ${ }^{21}$ III. Physikalisches Institut A, RWTH Aachen, Aachen, Germany \\ ${ }^{22}$ Physikalisches Institut, Universität Bonn, Bonn, Germany \\ ${ }^{23}$ Physikalisches Institut, Universität Freiburg, Freiburg, Germany \\ ${ }^{24}$ Institut für Physik, Universität Mainz, Mainz, Germany \\ ${ }^{25}$ Ludwig-Maximilians-Universität München, München, Germany \\ ${ }^{26}$ Fachbereich Physik, University of Wuppertal, Wuppertal, Germany \\ ${ }^{27}$ Panjab University, Chandigarh, India \\ ${ }^{28}$ Delhi University, Delhi, India \\ ${ }^{29}$ Tata Institute of Fundamental Research, Mumbai, India
}




\author{
${ }^{30}$ University College Dublin, Dublin, Ireland \\ ${ }^{31}$ Korea Detector Laboratory, Korea University, Seoul, Korea \\ ${ }^{32}$ Sung KyunKwan University, Suwon, Korea \\ ${ }^{33}$ CINVESTAV, Mexico City, Mexico \\ ${ }^{34}$ FOM-Institute NIKHEF and University of Amsterdam/NIKHEF, Amsterdam, The Netherlands \\ ${ }^{35}$ Radboud University Nijmegen/NIKHEF, Nijmegen, The Netherlands \\ ${ }^{36}$ Joint Institute for Nuclear Research, Dubna, Russia \\ ${ }^{37}$ Institute for Theoretical and Experimental Physics, Moscow, Russia \\ ${ }^{38}$ Moscow State University, Moscow, Russia \\ ${ }^{39}$ Institute for High Energy Physics, Protvino, Russia \\ ${ }^{40}$ Petersburg Nuclear Physics Institute, St. Petersburg, Russia \\ ${ }^{41}$ Lund University, Lund, Sweden, Royal Institute of Technology and Stockholm University, Stockholm, Sweden, \\ and Uppsala University, Uppsala, Sweden \\ ${ }^{42}$ Physik Institut der Universität Zürich, Zürich, Switzerland \\ ${ }^{43}$ Lancaster University, Lancaster, United Kingdom \\ ${ }^{44}$ Imperial College, London, United Kingdom \\ ${ }^{45}$ University of Manchester, Manchester, United Kingdom \\ ${ }^{46}$ University of Arizona, Tucson, Arizona 85721, USA \\ ${ }^{47}$ Lawrence Berkeley National Laboratory and University of California, Berkeley, California 94720, USA \\ ${ }^{48}$ California State University, Fresno, California 93740, USA \\ ${ }^{49}$ University of California, Riverside, California 92521, USA \\ ${ }^{50}$ Florida State University, Tallahassee, Florida 32306, USA \\ ${ }^{51}$ Fermi National Accelerator Laboratory, Batavia, Illinois 60510, USA \\ ${ }^{52}$ University of Illinois at Chicago, Chicago, Illinois 60607, USA \\ ${ }^{53}$ Northern Illinois University, DeKalb, Illinois 60115, USA \\ ${ }^{54}$ Northwestern University, Evanston, Illinois 60208, USA \\ ${ }^{55}$ Indiana University, Bloomington, Indiana 47405, USA \\ ${ }^{56}$ University of Notre Dame, Notre Dame, Indiana 46556, USA \\ ${ }^{57}$ Purdue University Calumet, Hammond, Indiana 46323, USA \\ ${ }^{58}$ Iowa State University, Ames, Iowa 50011, USA \\ ${ }^{59}$ University of Kansas, Lawrence, Kansas 66045, USA \\ ${ }^{60}$ Kansas State University, Manhattan, Kansas 66506, USA \\ ${ }^{61}$ Louisiana Tech University, Ruston, Louisiana 71272, USA \\ ${ }^{62}$ University of Maryland, College Park, Maryland 20742, USA \\ ${ }^{63}$ Boston University, Boston, Massachusetts 02215, USA \\ ${ }^{64}$ Northeastern University, Boston, Massachusetts 02115, USA \\ ${ }^{65}$ University of Michigan, Ann Arbor, Michigan 48109, USA \\ ${ }^{66}$ Michigan State University, East Lansing, Michigan 48824, USA \\ ${ }^{67}$ University of Mississippi, University, Mississippi 38677, USA \\ ${ }^{68}$ University of Nebraska, Lincoln, Nebraska 68588, USA \\ ${ }^{69}$ Princeton University, Princeton, New Jersey 08544, USA \\ ${ }^{70}$ State University of New York, Buffalo, New York 14260, USA \\ ${ }^{71}$ Columbia University, New York, New York 10027, USA \\ ${ }^{72}$ University of Rochester, Rochester, New York 14627, USA \\ ${ }^{73}$ State University of New York, Stony Brook, New York 11794, USA \\ ${ }^{74}$ Brookhaven National Laboratory, Upton, New York 11973, USA \\ ${ }^{75}$ Langston University, Langston, Oklahoma 73050, USA \\ ${ }^{76}$ University of Oklahoma, Norman, Oklahoma 73019, USA \\ ${ }^{77}$ Oklahoma State University, Stillwater, Oklahoma 74078, USA \\ ${ }^{78}$ Brown University, Providence, Rhode Island 02912, USA \\ ${ }^{79}$ University of Texas, Arlington, Texas 76019, USA \\ ${ }^{80}$ Southern Methodist University, Dallas, Texas 75275, USA \\ ${ }^{81}$ Rice University, Houston, Texas 77005, USA \\ ${ }^{82}$ University of Virginia, Charlottesville, Virginia 22901, USA \\ ${ }^{83}$ University of Washington, Seattle, Washington 98195, USA
} (Received 7 December 2007; published 9 April 2008)

We present the first measurement of the integrated forward-backward charge asymmetry in top-quarktop-antiquark pair $(t \bar{t})$ production in proton-antiproton $(p \bar{p})$ collisions in the lepton + jets final state. Using a $b$-jet tagging algorithm and kinematic reconstruction assuming $t \bar{t}+X$ production and decay, a sample of $0.9 \mathrm{fb}^{-1}$ of data, collected by the D0 experiment at the Fermilab Tevatron Collider, is used to 
measure the asymmetry for different jet multiplicities. The result is also used to set upper limits on $t \bar{t}+X$ production via a $Z^{\prime}$ resonance.

DOI: 10.1103/PhysRevLett.100.142002

At lowest order in quantum chromodynamics (QCD), the standard model (SM) predicts that the kinematic distributions in $p \bar{p} \rightarrow t \bar{t}+X$ production are charge symmetric. But this symmetry is accidental, as the initial $p \bar{p}$ state is not an eigenstate of charge conjugation. Next-to-leading order calculations predict forward-backward asymmetries of $(5-10) \%$ [1,2], but recent next-to-next-to-leading order calculations predict significant corrections for $t \bar{t}+$ jet production [3]. The asymmetry arises mainly from interference between contributions symmetric and antisymmetric under the exchange $t \leftrightarrow \bar{t}$ [1], e.g., between initial and final state gluon radiation in $q \bar{q} \rightarrow t \bar{t}+g$. It depends on the region of phase space being probed and, in particular, on the production of an additional jet [2]. The small asymmetries expected in the SM make this a sensitive probe for new physics [4].

A charge asymmetry in $p \bar{p} \rightarrow t \bar{t}+X$ can be observed as a forward-backward production asymmetry. The signed difference between the rapidities [5] of the $t$ and $\bar{t}$, $\Delta y \equiv y_{t}-y_{\bar{t}}$, reflects the asymmetry in $t \bar{t}$ production. We define the integrated charge asymmetry as $A_{\mathrm{fb}}=\left(N_{\mathrm{f}}-\right.$ $\left.N_{\mathrm{b}}\right) /\left(N_{\mathrm{f}}+N_{\mathrm{b}}\right)$, where $N_{\mathrm{f}}\left(N_{\mathrm{b}}\right)$ is the number of events with a positive (negative) $\Delta y$.

This Letter describes the first measurement of $A_{\mathrm{fb}}$ in $p \bar{p} \rightarrow t \bar{t}+X$ production. The $0.9 \mathrm{fb}^{-1}$ data sample used was collected at $\sqrt{s}=1.96 \mathrm{TeV}$ with the D0 detector [6], using triggers that required a jet and an electron or muon. In the lepton + jets final state of the $t \bar{t}$ system, one of the two $W$ bosons from the $t \bar{t}$ pair decays into hadronic jets and the other into leptons, yielding a signature of two $b$ jets, two light-flavor jets, an isolated lepton, and missing transverse energy $\left(\not{E}_{T}\right)$. This decay mode is well suited for this measurement, as it combines a large branching fraction ( $\sim 34 \%)$ with high signal purity, the latter a consequence of requiring an isolated electron or muon with large transverse momentum $\left(p_{T}\right)$. The main background is from $W+$ jets and multijet production. This channel allows accurate reconstruction of the $t$ and $\bar{t}$ directions in the collision rest frame, and the charge of the electron or muon distinguishes between the $t$ and $\bar{t}$ quarks.

The dependence of $A_{\mathrm{fb}}$ on the region of phase space, as calculated by the MC@NLO event generator [7], shows that acceptance can strongly affect the asymmetry. In particular, the largest acceptance effects are due to the requirement of $\geq 4$ jets above some $p_{T}$ threshold, and the generated asymmetry varies from $+8 \%$ to $-3 \%$ as a function of the fourth-highest particle jet $p_{T}$ [8]. To facilitate comparison with theory, the analysis is therefore designed to have an acceptance which can be described simply. Event selection is limited to either (i) selections
PACS numbers: 12.38.Qk, 12.60.-i, 13.85.-t, 13.87.Ce

on directions and momenta that can be described at the particle level (which refers to produced particles before they interact with material in the detector) or (ii) criteria with high signal efficiency, so that their impact on the region of acceptance is negligible. In addition, the observable quantity and the fitting procedure are chosen to ensure that all events have the same weight in determining the asymmetry.

The measurement is not corrected for acceptance and reconstruction effects, but a prescription provides the acceptance at the particle level. Reconstruction effects are also accommodated at the particle level by defining the asymmetry as a function of the generated $|\Delta y|$ :

$$
A_{\mathrm{fb}}(|\Delta y|)=\frac{g(|\Delta y|)-g(-|\Delta y|)}{g(|\Delta y|)+g(-|\Delta y|)}
$$

where $g$ is the probability density for $\Delta y$ within the acceptance. This asymmetry can be folded with the "geometric dilution" $\mathcal{D}$, which is described later:

$$
A_{\mathrm{fb}}^{\mathrm{pred}}=\int_{0}^{\infty} A_{\mathrm{fb}}(\Delta y) \mathcal{D}(\Delta y)[g(\Delta y)+g(-\Delta y)] d \Delta y .
$$

This procedure yields the predictions in Table I. The values are smaller than those of Refs. [1,2], because of the inclusion of jet acceptance and dilution.

We select events with at least four jets reconstructed using a cone algorithm [9] with an angular radius $\mathcal{R}=0.5$ (in rapidity and azimuthal angle). All jets must have $p_{T}>$ $20 \mathrm{GeV}$ and pseudorapidity (relative to the reconstructed primary vertex) $|\eta|<2.5$. The leading jet must have $p_{T}>$ $35 \mathrm{GeV}$. Events are required to have $\not E_{T}>15 \mathrm{GeV}$ and exactly one isolated electron with $p_{T}>15 \mathrm{GeV}$ and $|\eta|<$ 1.1 or one isolated muon with $p_{T}>18 \mathrm{GeV}$ and $|\eta|<2.0$. More details on lepton identification and trigger requirements are given in Ref. [10]. Events in which the lepton momentum is mismeasured are suppressed by requiring that the direction of the $\mathbb{E}_{T}$ not be along or opposite the azimuth of the lepton. To enhance the signal, at least one of the jets is required to be identified as originating from a long-lived $b$ hadron by a $b$-jet tagging algorithm [11] which relies on the presence and characteristics of a sec-

TABLE I. Detector level predictions based on MC@NLO.

\begin{tabular}{lr}
\hline \hline$N_{\text {jet }}$ & $A_{\mathrm{fb}}^{\text {pred }}($ in $\%)$ \\
$\geq 4$ & $0.8 \pm 0.2($ stat $) \pm 1.0($ accept $) \pm 0.0($ dilution $)$ \\
4 & $2.3 \pm 0.2($ stat $) \pm 1.0($ accept $) \pm 0.1$ (dilution) \\
$\geq 5$ & $-4.9 \pm 0.4($ stat $) \pm 1.0($ accept $) \pm 0.2$ (dilution) \\
\hline \hline
\end{tabular}


ondary vertex and tracks with high impact parameter inside the jet.

The top-quark pair is reconstructed using a kinematic fitter [12], which varies the four-momenta of the detected objects within their resolutions and minimizes a $\chi^{2}$ statistic, constraining both $W$ boson masses to exactly $80.4 \mathrm{GeV}$ and top quark masses to exactly $170 \mathrm{GeV}$. The $b$-tagged jet of highest $p_{T}$ and the three remaining jets with highest $p_{T}$ are used in the fit. The $b$-tagging information is used to reduce the number of jet-parton assignments considered in the fit. Only events in which the kinematic fit converges are used, and for each event only the reconstruction with the lowest $\chi^{2}$ is retained.

The selection criteria can be approximated by simple cuts on particle-level momenta without changing the generated asymmetry by more than $2 \%$ (absolute). This is verified using several simulated samples with generated asymmetries and particle jets clustered using the PXCONE algorithm [13] ("E" scheme and $\mathcal{R}=0.5$ ). The particle jet cuts are $p_{T}>21 \mathrm{GeV}$ and $|\eta|<2.5$, with the additional requirement on the leading particle jet $p_{T}>35 \mathrm{GeV}$ and the lepton requirements detailed above. Systematic uncertainties on jet energy calibration introduce possible shifts of the particle jet thresholds. The shifts are ${ }_{-1.5}^{+1.3} \mathrm{GeV}$ for the leading jet and ${ }_{-1.3}^{+1.2} \mathrm{GeV}$ for the other jets, for \pm 1 standard deviation $(s d)$ changes in the jet energy calibration. The resulting changes in the asymmetry predicted using MC@NLO are of the order of 0.5\%. The effect of all other selections on the asymmetry is negligible. The predictions in Table I use a more complete description of the acceptance based on efficiencies factorized in $p_{T}$ and $\eta$, accurate to $<1 \%$ (absolute).

Misreconstructing the sign of $\Delta y$ dilutes the asymmetry. Such dilution can arise from misidentifying lepton charge or from misreconstructing event geometry. The rate for misidentification of lepton charge is taken from the signal simulation and verified using leptonic $Z$ boson decays in data. False production asymmetries arising from asymmetries in the rate for misidentification of lepton charge are negligible owing to the frequent reversal of the D0 solenoid and toroid polarities.

The dilution $\mathcal{D}$ depends mainly on $|\Delta y|$. It is defined as $\mathcal{D}=2 P-1$, where $P$ is the probability of reconstructing the correct sign of $\Delta y$. It is obtained from $t \bar{t}+X$ events generated with PYTHIA [14] and passed through a GEANTbased simulation [15] of the D0 detector, and is parametrized as

$$
\mathcal{D}(|\Delta y|)=c_{0} \ln \left(1+c_{1}|\Delta y|+c_{2}|\Delta y|^{2}\right),
$$

with the parameters given in Table II [8].

As this measurement is integrated in $|\Delta y|$, the dependence of the dilution on $|\Delta y|$ introduces a model dependence into any correction from observed asymmetry $\left(A_{\mathrm{fb}}^{\mathrm{obs}}\right)$ to a particle-level asymmetry. Such a correction factor would depend not only on the model's $|\Delta y|$ distribution,
TABLE II. Parameters of the dilution. The $\pm 1 s d$ values include both statistical and systematic uncertainties.

\begin{tabular}{lccr}
\hline \hline Variation & $c_{0}$ & $c_{1}$ & $c_{2}$ \\
\hline$N_{\text {jet }} \geq 4$ & 0.262 & 14.6 & -1.5 \\
$+1 s d$ variation & 0.229 & 20.3 & 1.2 \\
$-1 s d$ variation & 0.289 & 11.4 & -2.2 \\
$N_{\text {jet }}=4$ & 0.251 & 17.6 & -1.4 \\
$+1 s d$ variation & 0.201 & 30.3 & 7.7 \\
$-1 s d$ variation & 0.293 & 11.6 & -2.3 \\
$N_{\text {jet }} \geq 5$ & 0.254 & 9.6 & 0 \\
$+1 s d$ variation & 0.206 & 17.4 & 2.4 \\
$-1 s d$ variation & 0.358 & 5.0 & -0.9 \\
\hline \hline
\end{tabular}

but also on its prediction of $A_{\mathrm{fb}}(|\Delta y|)$. Furthermore, such a correction would be sensitive to small new physics components of the selected sample. We therefore present a measurement uncorrected for reconstruction effects and provide the reader with a parametrization of $\mathcal{D}$ that describes these effects, to be applied to any model.

The dilution depends weakly on other variables correlated with $A_{\mathrm{fb}}$, such as the number of jets. This possible bias is included in the systematic uncertainties. Nonstandard production mechanisms can affect reconstruction quality, primarily due to changes in the momenta of the top quarks. By studying extreme cases, we find that when comparing nonstandard $t \bar{t}+X$ production to data an additional $15 \%$ relative uncertainty on $A_{\mathrm{fb}}$ is needed.

The main background is from $W+$ jets production. To estimate it, we define a likelihood discriminant $\mathcal{L}$ using variables that are well described in our simulation, provide separation between signal and $W+$ jets background, and do not bias $|\Delta y|$ for the selected signal. Discrimination is based mainly on the $p_{T}$ of the leading $b$-tagged jet and the $\chi^{2}$ statistic from the kinematic fit.

The next largest background after $W+$ jets is from multijet production, where a jet mimics an isolated electron or muon. Following the procedure described in Ref. [10], the distributions in likelihood discriminant and reconstructed asymmetry for this background are derived from samples of data that fail lepton identification. The normalization of this background is estimated from the size of those samples and the large difference in efficiencies of lepton identification for true and false leptons. The effects of additional background sources not considered explicitly

TABLE III. Number of selected events and fit results in data.

\begin{tabular}{lccc}
\hline \hline & $\geq 4$ Jets & 4 Jets & $\geq 5$ Jets \\
No. Events & 376 & 308 & 68 \\
\hline$t \bar{t}+X$ & $266_{-22}^{+23}$ & $214 \pm 20$ & $54_{-12}^{+10}$ \\
$W+$ jets & $70 \pm 21$ & $61_{-18}^{+19}$ & $7_{-5}^{+11}$ \\
Multijets & $40 \pm 4$ & $32.7_{-3.3}^{+3.5}$ & $7.1_{-1.5}^{+1.6}$ \\
$A_{\mathrm{fb}}$ & $(12 \pm 8) \%$ & $(19 \pm 9) \%$ & $\left(-16_{-17}^{+15}\right) \%$ \\
\hline \hline
\end{tabular}



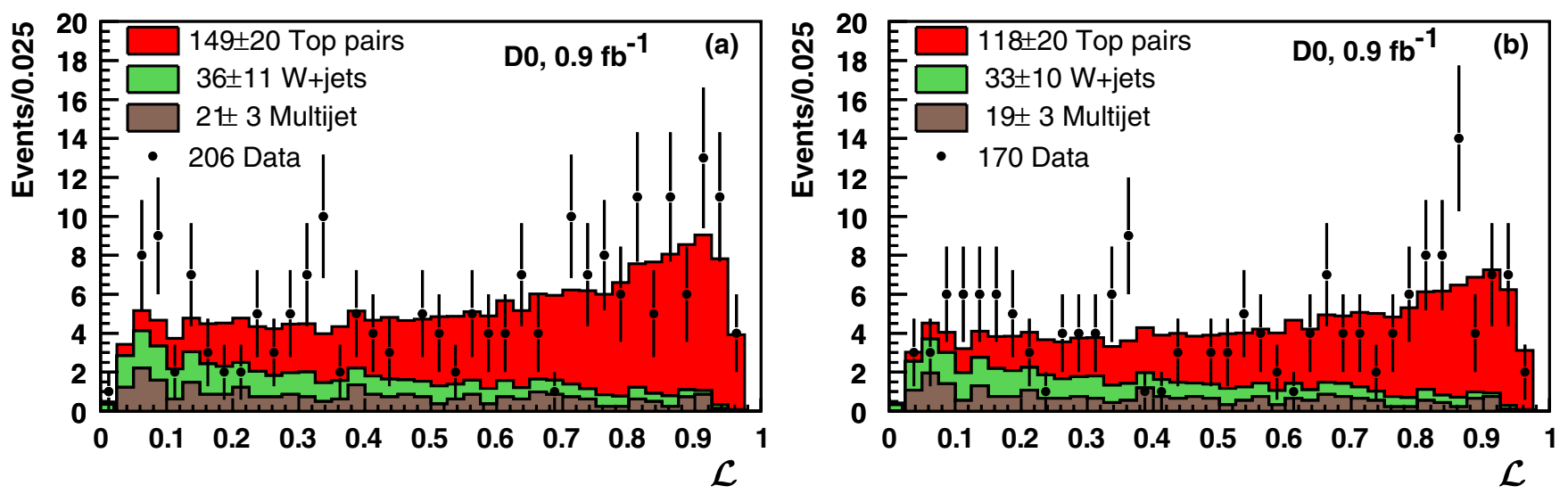

FIG. 1 (color online). Comparison of data for $\geq 4$ jets with the fitted model as a function of $\mathcal{L}$ for events reconstructed (a) as forward $\left(\Delta y_{\text {reco }}>0\right)$ and (b) as backward $\left(\Delta y_{\text {reco }}<0\right)$. The number of events from each source is listed with its statistical uncertainty.

in extracting $A_{\mathrm{fb}}$, namely $Z+$ jets, single top quark, and diboson production, are evaluated using ensembles of simulated data sets and found negligible.

The sample composition and $A_{\mathrm{fb}}$ are extracted from a simultaneous maximum-likelihood fit to data of a sum of contributions to $\mathcal{L}$ and to the sign of the reconstructed $\Delta y$ $\left(\Delta y_{\text {reco }}\right)$ from forward signal, backward signal, $W+$ jets, and multijet production. Both signal contributions are generated with PYTHIA, have the same distribution in $\mathcal{L}$, and differ only in their being reconstructed as either forward or backward. The $W+$ jets contribution is generated with ALPGEN [16] interfaced to PYTHIA and has its own reconstructed asymmetry. Although $W$ boson production is inherently asymmetric, the kinematic reconstruction to the $t \bar{t}+X$ hypothesis reduces its reconstructed asymmetry to $[4.4 \pm 1.6$ (stat) $] \%$. The multijet contribution is derived from data, as described above. The fitted parameters are shown in Table III. Correlations between the asymmetry and the other parameters are $<10 \%$. In Fig. 1 we compare the fitted distributions to data for events with $\geq 4$ jets.

The dominant sources of systematic uncertainty for the measured asymmetry are the relative jet energy calibration between data and simulation $( \pm 0.5 \%)$, the asymmetry reconstructed in $W+$ jets events $( \pm 0.4 \%)$, and the modeling of additional interactions during a single $p \bar{p}$ bunch crossing $( \pm 0.4 \%)$. The total systematic uncertainty for the asymmetry is $\pm 1 \%$, which is negligible compared with the statistical uncertainty.

We check the simulation of the production asymmetry, and of the asymmetry reconstructed under the $t \bar{t}+X$ hypothesis in the $W+$ jets background, by repeating the analysis in a sample enriched in $W+$ jets events. The selection criteria for this sample are identical to the main analysis, except that we veto on any $b$ tags. Both the reconstructed $A_{\mathrm{fb}},[-2 \pm 5(\mathrm{stat})] \%$, and the forwardbackward lepton asymmetry, $[13 \pm 5$ (stat) $] \%$, are consistent with expectations $([3.7 \pm 1.5$ (stat) $] \%$ and $[11.6 \pm$ 1.5 (stat) $] \%$, respectively).
To demonstrate the measurement's sensitivity to new physics, we examine $t \bar{t}$ production via neutral gauge bosons $\left(Z^{\prime}\right)$ that are heavy enough to decay to on-shell top and antitop quarks. Direct searches have placed limits on $t \bar{t}$ production via a heavy narrow resonance [17], while the asymmetry in $t \bar{t}$ production may be sensitive to production via both narrow and wide resonances. The $Z^{\prime} \rightarrow t \bar{t}$ channel is of interest in models with a "leptophobic" $Z$ ' that decays dominantly to quarks. We study the scenario where the coupling between the $Z^{\prime}$ boson and quarks is proportional to that between the $Z$ boson and quarks, and interference effects with SM $t \bar{t}$ production are negligible. Using PYTHIA we simulate $t \bar{t}$ production via $Z^{\prime}$ resonances with decay rates chosen to yield narrow resonances as in Ref. [17], and find large positive asymmetries [(13-35)\%], which are a consequence of the predominantly left-handed decays. We predict the distribution of $A_{\mathrm{fb}}$ as a function of the fraction $(f)$ of $t \bar{t}$ events produced via a $Z^{\prime}$ resonance of a particular mass from ensembles of simulated data sets. We use the procedure of Ref. [18] to arrive at the 95\% C.L. limits shown in Fig. 2. The expected limits, and their variations 1 and 2 standard deviations away due to statistical fluctua-

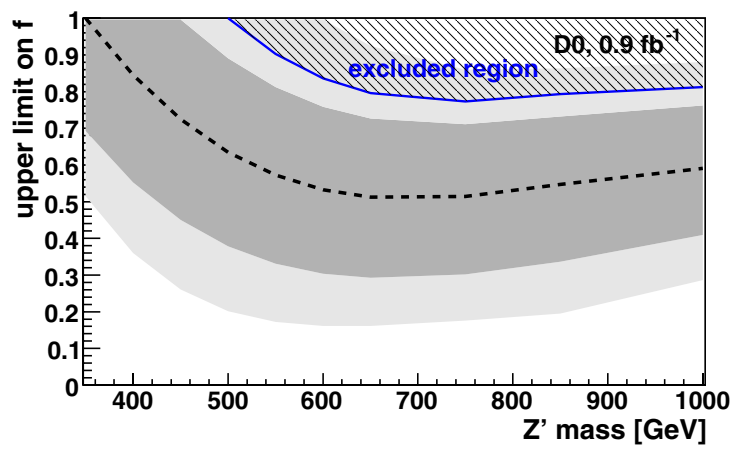

FIG. 2 (color online). Limits on $f$ as a function of the $Z^{\prime}$ mass. Solid curve and hatching for the observed limits and the excluded region, dashed curve and shaded bands for the expected limits and their statistical variations. 
tions, are determined from ensembles with no $Z^{\prime}$ contribution. These limits can be applied to wide $Z^{\prime}$ resonances by averaging over the distribution of $Z^{\prime}$ mass.

In summary, we present the first measurement of the integrated forward-backward charge asymmetry in $t \bar{t}+X$ production. We find that acceptance affects the asymmetry and must be specified as above, and that corrections for reconstruction effects are too model dependent to be of use. We observe an uncorrected asymmetry of $A_{\mathrm{fb}}^{\text {obs }}=[12 \pm$ 8 (stat) \pm 1 (syst) $] \%$ for $t \bar{t}+X$ events with $\geq 4$ jets that are within our acceptance, and we provide a dilution function [Eq. (3)] that can be applied to any model [through Eq. (2)]. For events with only four jets and for those with $\geq 5$ jets, we find $A_{\mathrm{fb}}^{\text {obs }}=[19 \pm 9$ (stat) $\pm 2($ syst $)] \%$ and $A_{\mathrm{fb}}^{\text {obs }}=$ $\left[-16_{-17}^{+15}\right.$ (stat) \pm 3 (syst) $] \%$, respectively, where most of the systematic uncertainty is from migrations of events between the two subsamples. The measured asymmetries are consistent with the MC@NLO predictions for SM production.

We thank the staffs at Fermilab and collaborating institutions, and acknowledge support from the DOE and NSF (USA); CEA and CNRS/IN2P3 (France); FASI, Rosatom, and RFBR (Russia); CAPES, CNPq, FAPERJ, FAPESP, and FUNDUNESP (Brazil); DAE and DST (India); Colciencias (Colombia); CONACyT (Mexico); KRF and KOSEF (Korea); CONICET and UBACyT (Argentina); FOM (The Netherlands); Science and Technology Facilities Council (United Kingdom); MSMT and GACR (Czech Republic); CRC Program, CFI, NSERC and WestGrid Project (Canada); BMBF and DFG (Germany); SFI (Ireland); The Swedish Research Council (Sweden); CAS and CNSF (China); Alexander von Humboldt Foundation; and the Marie Curie Program.

\footnotetext{
*Visitor from Augustana College, Sioux Falls, SD, USA

${ }^{\dagger}$ Visitor from The University of Liverpool, Liverpool, UK.

${ }^{\ddagger}$ Deceased.

${ }^{\S}$ Visitor from II. Physikalisches Institut, Georg-AugustUniversity, Göttingen, Germany.

"Visitor from ICN-UNAM, Mexico City, Mexico.
}

"Visitor from Helsinki Institute of Physics, Helsinki, Finland.

**Visitor from Universität Zürich, Zürich, Switzerland.

[1] J.H. Kühn and G. Rodrigo, Phys. Rev. D 59, 054017 (1999).

[2] M. T. Bowen, S. D. Ellis, and D. Rainwater, Phys. Rev. D 73, 014008 (2006).

[3] S. Dittmaier P. Uwer, and S. Weinzierl, Phys. Rev. Lett. 98, 262002 (2007).

[4] For example, O. Antuñano, J.H. Kühn, and G. Rodrigo, Phys. Rev. D 77, 014003 (2008).

[5] Rapidity $y$ and pseudorapidity $\eta$ are defined as functions of the polar angle $\theta$ as $y(\theta, \beta) \equiv \frac{1}{2} \ln [(1+\beta \cos \theta) /(1-$ $\beta \cos \theta)] ; \eta(\theta) \equiv y(\theta, 1)$, where $\beta$ is the ratio of a particle's momentum to its energy.

[6] V. M. Abazov et al. (D0 Collaboration), Nucl. Instrum. Methods Phys. Res., Sect. A 565, 463 (2006).

[7] S. Frixione and B. R. Webber, J. High Energy Phys. 06 (2002) 29;S. Frixione et al., ibid. 08 (2003) 7.

[8] See EPAPS Document No. E-PRLTAO-100-059814 for additional plots. For more information on EPAPS, see http://www.aip.org/pubservs/epaps.html.

[9] G. C. Blazey et al., in Proceedings of the Workshop: QCD and Weak Boson Physics in Run II, edited by U. Baur, R. K. Ellis, and D. Zeppenfeld (Fermilab Report No. Fermilab-Pub-00/297, 2000).

[10] V. M. Abazov et al. (D0 Collaboration), Phys. Rev. D 76, 092007 (2007).

[11] T. Scanlon, Ph.D. thesis, University of London (Fermilab Report No. Fermilab-Thesis-2006-43, 2006).

[12] S. Snyder, Ph.D. thesis, State University of New York at Stony Brook, 1995.

[13] C. Adloff et al. (H1 Collaboration), Nucl. Phys. B545, 3 (1999).

[14] T. Sjöstrand et al., Comput. Phys. Commun. 135, 238 (2001).

[15] R. Brun and F. Carminati, CERN Program Library Long Writeup Report No. W5013, 1993 (unpublished).

[16] M. L. Mangano et al., J. High Energy Phys. 07 (2003) 1; S. Höche et al., arXiv:hep-ph/0602031.

[17] V. M. Abazov et al. (D0 Collaboration), Phys. Rev. Lett. 92, 221801 (2004); T. Aaltonen et al. (CDF Collaboration), arXiv:0709.0705.

[18] G. J. Feldman and R. D. Cousins, Phys. Rev. D 57, 3873 (1998). 